\title{
Central aortic blood pressure and augmentation index during normal pregnancy
}

\author{
Mika Fujime ${ }^{1}$, Takuji Tomimatsu ${ }^{1}$, Yuko Okaue ${ }^{2}$, Shinsuke Koyama ${ }^{1}$, Takeshi Kanagawa ${ }^{1}$, Takeshi Taniguchi ${ }^{2}$ \\ and Tadashi Kimura ${ }^{1}$
}

The current study tested the hypothesis that pregnancy-related changes are more pronounced in central hemodynamics, and both central aortic systolic blood pressure (cSBP) and augmentation index (Alx) are independent from brachial systolic blood pressure (bSBP) in normal pregnant subjects. In 830 healthy pregnant women from 12 to 36 weeks gestation, we measured CSBP and Alx-75 (Alx at heart rate of 75 beats per minute) non-invasively by pulse waveforms of the radial artery using an automated applanation tonometric system. In 69 pregnant women, we recorded these data longitudinally. cSBP and Alx-75 significantly declined during pregnancy, reaching its nadir in mid-pregnancy and rising towards term. Pregnancy-related changes were more pronounced in Alx-75 compared with CSBP, but less evident in bSBP. Alx-75, but not cSBP, was independent from bSBP throughout pregnancy. CSBP and Alx-75, but not bSBP, were significantly increased in healthy pregnant women older than 35 years. This study established normal values for pulse wave analysis parameters throughout pregnancy, and indicated that pulse wave analysis might offer additional and independent information about maternal arterial compliance to conventional brachial blood pressure measurements. These data may be used as the basis for further investigation into the role of pulse wave analysis in the assessment, management and prediction of disorders, which might interfere with pregnancy-related cardiovascular adaptations.

Hypertension Research (2012) 35, 633-638; doi:10.1038/hr.2012.1; published online 2 February 2012

Keywords: arterial stiffness; pregnancy; pulse wave analysis

\section{INTRODUCTION}

The measurement of brachial blood pressure has long been an integral part of antenatal care, and elevated brachial blood pressure has been considered to be the hallmark for diagnosis of preeclampsia. However, it has been widely recognized that hypertension itself is not necessarily related to adverse maternal and fetal outcomes except for an increased risk of maternal intracranial hemorrhage, and additional markers to establish or rule out imminent adverse outcomes has been really needed for timely intervention. ${ }^{1}$

Although it has been firmly established that brachial blood pressure predicts future cardiovascular disease in the non-pregnant population, recent accumulative evidence has indicated that arterial stiffness, expressed as central aortic systolic blood pressure (cSBP) or augmentation index (AIx), is more closely or independently correlated with future cardiovascular events. ${ }^{2-5}$ Using applanation tonometry, cSBP and AIx can be assessed non-invasively and accurately by pulse wave analysis of peripheral arteries (such as the radial artery), and these markers have been increasingly incorporated into clinical practice of cardiovascular as well as other areas of medicine. ${ }^{6-11}$

In pregnant women, recent studies have reported increased arterial stiffness in women with preeclampsia. ${ }^{12-16}$ It has also been suggested that AIx measured at 11-14 weeks can predict subsequent develop- ment of preeclampsia. ${ }^{17}$ In addition, women with a history of earlyonset preeclampsia and intrauterine growth restriction were reported to exhibit increased AIx even 6 to 24 months postpartum. ${ }^{18}$ Although two recent studies addressed normal values of CSBP and AIx throughout pregnancy showing its nadir in mid-pregnancy, ${ }^{19,20}$ their physiological aspects in healthy pregnant individuals, such as their relationships to brachial blood pressure, are currently unknown.

In this study, we hypothesized that pregnancy-related changes are more pronounced in central hemodynamics, and both cSBP and AIx are independent from brachial blood pressure during pregnancy. In the largest cohort of healthy pregnant individuals, the aim of this study is to test this hypothesis, to provide reference values for cSBP and AIx, and to explore their physiological meanings using a userfriendly, operator-independent and automated device.

\section{METHODS}

Subjects

We conducted a cross sectional study involving 830 Asian women in normal singleton pregnancies from 12 to 36 weeks gestation, at Osaka University medical hospital and Taniguchi hospital from January 2009 to May 2010. Among the women who had more than one measurement during our study period, one measurement from each woman was randomly selected for analysis. 
Of 830 healthy pregnant women, 255 were recruited in the first trimester (1214 weeks), 230 in the second trimester (17-20 weeks), 218 in the late second trimester (23-27 weeks) and 127 in the third trimester (34-36 weeks). In 69 pregnant women, we recorded data of cSBP and AIx longitudinally in the first (12-14 weeks), the late second (23-27 weeks) and the third (34-36 weeks) trimesters, at a few days after delivery and 4 weeks postpartum.

Maternal characteristics, including age, parity and gestational age were recorded. In all pregnant women in this study, gestational age was confirmed by ultrasonographic examination before 12 weeks gestation. In this study, we only examined pregnant women who had uncomplicated pregnancies. The subjects with a history of chronic hypertension, diabetes mellitus, any previous or concomitant cardiovascular disease, cardiac arrhythmias, multiple pregnancy, preeclampsia, Basedow's disease, renal disorder and immune disorders were excluded from the study.

The study had official approval from the Institutional Ethics Committee of Osaka University, and written informed consent was also obtained from all subjects.

\section{Pulse wave analysis}

Arterial pulse waveforms of the right radial artery were measured noninvasively by an automated applanation tonometric system (HEM-9000AI; Omron Healthcare, Kyoto, Japan). Using this device, we could measure various hemodynamic parameters such as cSBP, AIx and AIx-75 (AIx at heart rate of 75 beats per minute). The measurement time was about $1 \mathrm{~min}$, and the device was capable of taking measurements in the sitting position. So, it was adapted for all pregnant women without any difficulties. The methodology for measuring radial pulse waveform by this device has been described in detail in previous manuscripts. ${ }^{21-23}$ In brief, when the tonometry sensor unit with 40 microtransducer elements is placed on the subject's wrist, and one of these elements is automatically selected to obtain optimal radial arterial pressure waveform. As shown in Figure 1, the systolic part of the radial arterial waveform was

\section{a Aortic pulse waveform}

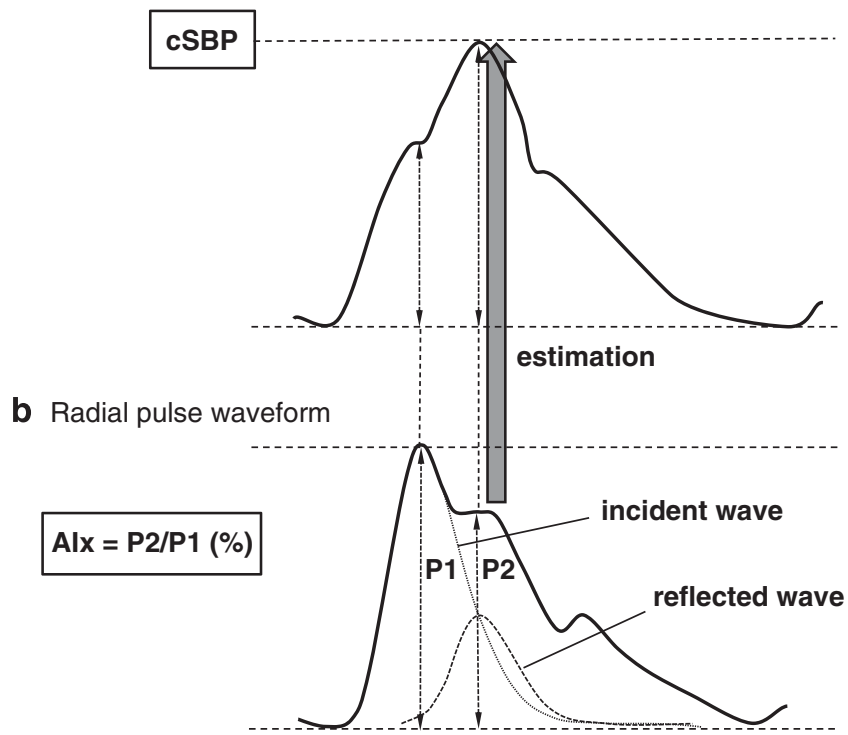

Figure 1 Central aortic systolic blood pressure (CSBP) and definition of the augmentation index (Alx). P1 indicates the amplitude of the early systolic peak pressure, P2 the amplitude of the late systolic peak pressure. As P2 is generated by an interaction between the incident pulse wave and the reflected pulse wave, P2 is thought to represent the systemic arterial elasticity, which is arterial stiffness. The radial Alx is defined as the ratio of $\mathrm{P} 2$ to $\mathrm{P} 1$. As there is a linear relationship between heart rate and Alx, the Alx is standardized to a heart rate of 75 b.p.m. (Alx-75). The pressure pulse waveform at the aorta (a) is markedly different from that at the radial artery (b). CSBP was estimated by the value of P2, which has been reported to show a close linear correlation $(r=0.95)$ with the invasively measured intraaortic systolic pressure. ${ }^{22}$ characterized by two pressure peaks, the early systolic peak pressure (P1) and the late systolic peak pressure (P2). As P2 is generated by an interaction between the incident pulse wave and the reflected pulse wave, $\mathrm{P} 2$ is thought to represent the systemic arterial elasticity, that is, arterial stiffness. The software program incorporated into the HEM-9000AI identifies P2 automatically from the second maximum of the fourth derivative of the pulse waveform. The radial AIx was defined as the ratio of the height of $\mathrm{P} 2$ to that of $\mathrm{P} 1$ : $\mathrm{AI}=(\mathrm{P} 2 / \mathrm{P} 1) \times 100$ (\%) (Figure 1). As there is a linear relationship between the heart rate and AIx, the AIx is usually standardized to a heart rate of 75 b.p.m. (AIx-75). The pressure pulse waveform at the aorta is markedly different from that at peripheral arteries such as radial and brachial arteries. As shown in Figure 1, maximal aortic systolic pressure usually coincides with the second systolic peak, but in the peripheral arteries it usually coincides with the first systolic peak. Therefore, it has been thought that the CSBP reflects systemic arterial stiffness as well as left ventricular ejection, whereas peripheral systolic blood pressure mainly reflects left ventricular ejection. The cSBP was estimated by the value of
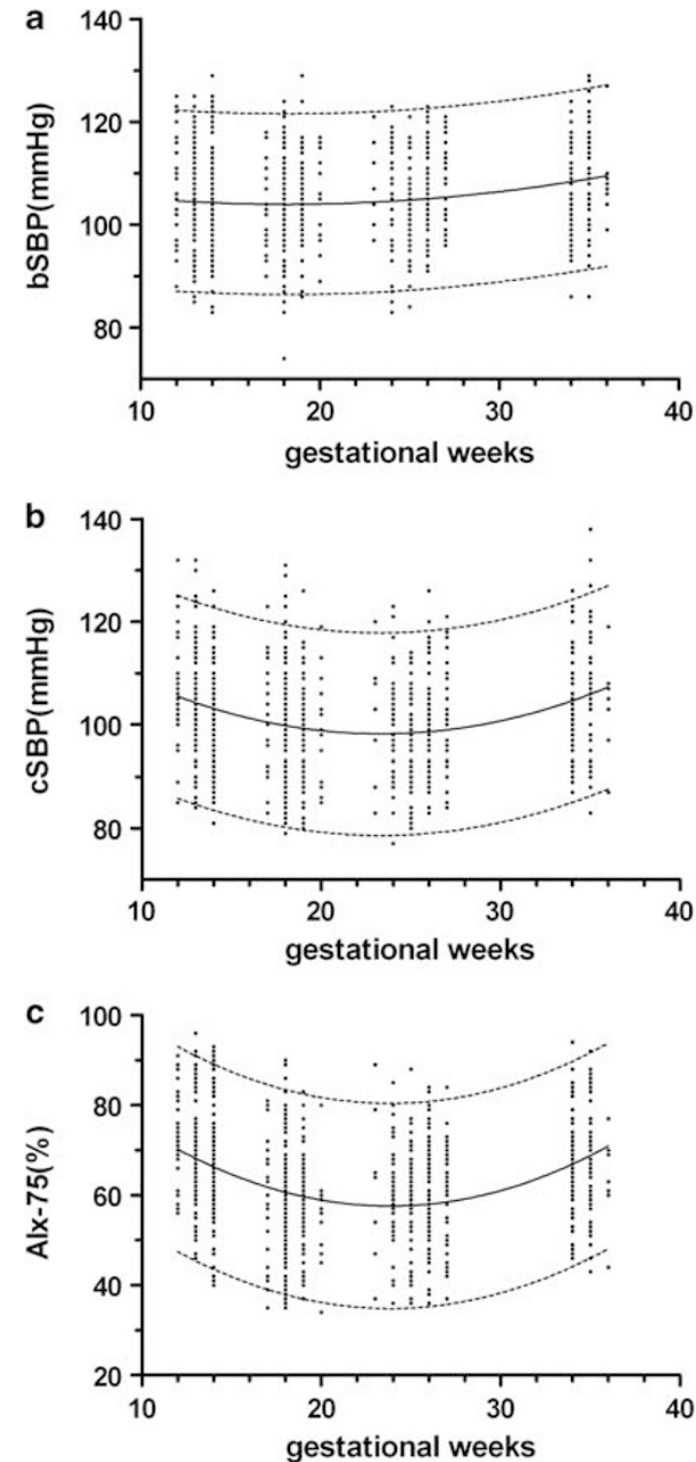

Figure 2 The trends and values of brachial systolic blood pressure (bSBP) (a), central aortic systolic blood pressure (cSBP) (b) and augmentation index at heart rate of 75 b.p.m. (Alx-75) (c). With gestation in 830 normal healthy pregnancies are shown. Each panel shows the regression line and the $95 \%$ prediction bands for mean and individual raw values of the Alxes. bSBP $=109.8-0.6342 \times \mathrm{GA}+0.01742 \times \mathrm{GA}^{2} . \quad \mathrm{cSBP}=129.0-2.640 \times$ $\mathrm{GA}+0.05659 \times \mathrm{GA}^{2}$. Alx-75 $(\%)=108.9-4.298 \times \mathrm{GA}+0.09013 \times \mathrm{GA}^{2}$. 
P2, which has been reported to show a close linear correlation $(r=0.95)$ with the invasively measured intra-aortic systolic pressure. ${ }^{22}$ Radial AIx has been reported to show a close correlation with aortic $\mathrm{AIx}$ ( $r=0.81$ to 0.96 ), thus suggesting a similar physiological significance between aortic and radial $\mathrm{AI}^{22-26}$ Continuous steady-state 15-sec data were recorded for each subject, and the mean values for all pulses were used in the analysis. The coefficients of variation of intra- and inter-observer measurements for AIx and CSBP using this device were reported to be around 2 to $4 \%,{ }^{21,25}$ indicating good reproducibility. As we first applied this system to the pregnant women in this study, its validity in pregnant individuals had not yet been demonstrated.

Brachial blood pressure was measured simultaneously in the opposite arm with an oscillometric device incorporated in HEM-9000AI. Brachial systolic blood pressure (bSBP) was defined by the first Korotkoff sound.

\section{Statistical analysis}

Data were expressed as mean \pm s.d. or as median with 95th percentile values. The effect of gestational age on bSBP and pulse wave analysis parameters (cSBP

Table 1 The median and 95th percentile values for bSBP, cSBP and Alx-75 in each trimester

\begin{tabular}{|c|c|c|c|c|c|c|c|}
\hline \multirow[b]{2}{*}{$G A$} & \multirow[b]{2}{*}{$n$} & \multicolumn{2}{|c|}{$b S B P(m m H g)$} & \multicolumn{2}{|c|}{$c S B P(m m ~ H g)$} & \multicolumn{2}{|c|}{ Alx-75 (\%) } \\
\hline & & Median & $95 \%$ ile & Median & $95 \%$ \%ile & Median & $95 \%$ ile \\
\hline $12-14$ & 255 & 104 & 121 & 103 & 123 & 69.0 & 89.0 \\
\hline $17-20$ & 230 & 104 & 118 & $99 *$ & 118 & $57.0^{*}$ & 79.5 \\
\hline $23-27$ & 218 & 105 & 120 & $99 *$ & 117 & $61.0 *$ & 79.0 \\
\hline $34-36$ & 127 & $108 *$ & 124 & 105 & 122 & 67.0 & 86.6 \\
\hline
\end{tabular}

Abbreviations: Alx, augmentation index; bSBP, brachial systolic blood pressure; cSBP, central aortic systolic blood pressure; GA, gestational age.

The median and 95th percentile (\%ile) values for bSBP, cSBP and Alx-75 in each trimester.

*Significant difference from first trimester $(12-14$ weeks gestation) values $(P<0.05)$. and AIx-75) was examined using regression analysis for continuous variables. Means of bSBP, cSBP and AIx-75 were analyzed throughout pregnancy with the use of one-way analysis of variance with the Bonferroni's post hoc test. Pearson's correlation coefficient $(r)$ was used to determine the correlation between bSBP and CSBP, or between bSBP and AIx-75, and between maternal height, bodymass index and bSBP, cSBP and AIx-75. For the longitudinal data, one-way analysis of variance with repeated measures, followed by the Bonferroni's post hoc test was used. Values of $P<0.05$ were considered to be statistically significant. All data were analyzed using GraphPad Prism 5 (GraphPad, San Diego, CA, USA).

\section{RESULTS}

In our cohort of 830 women in normal singleton pregnancy, recordings were successfully carried out without any difficulties. The mean maternal age was $31.0 \pm 5.0$ years, and nulliparity was $47.8 \%$. The mean maternal height, pre-pregnancy weight and pre-pregnancy bodymass index were $158.1 \pm 5.6 \mathrm{~cm}, 52.9 \pm 10.0 \mathrm{~kg}$ and $21.1 \pm 3.6 \mathrm{~kg} \mathrm{~m}^{-2}$, respectively. In our cohort, current smokers and pregnant women who quit smoking after their last menstrual period were 9.1 and $12.2 \%$, respectively. Smoking status was not associated with bSBP, cSBP and AIx-75 levels. Maternal height showed negative association with cSBP $(r=-0.15, P=0.046)$ and AIx-75 $(r=-0.26, P<0.001)$, but not with bSBP $(r=-0.05, P=0.52)$. In addition, maternal pre-pregnancy bodymass index showed positive association with bSBP $(r=0.29, P<0.001)$, cSBP $(r=0.31, P<0.001)$ and AIx-75 $(r=0.20, P=0.01)$.

The changes in bSBP, cSBP and AIx-75 according to gestational weeks are presented in Figure 2. bSBP did not change from first to second trimesters, and tended to increase toward term. In contrast, cSBP and AIx-75 showed similar changes; they reached nadir around midpregnancy, and increased to first-trimester levels toward term. The median and 95th percentile values for bSBP, cSBP and AIx-75 in each trimester are given in Table 1. a

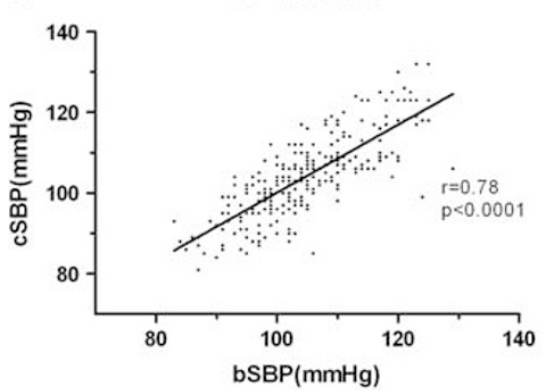

d

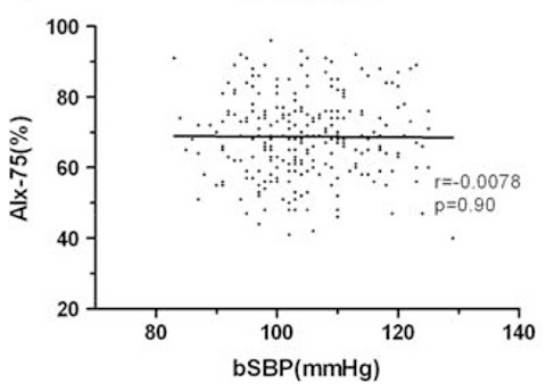

b

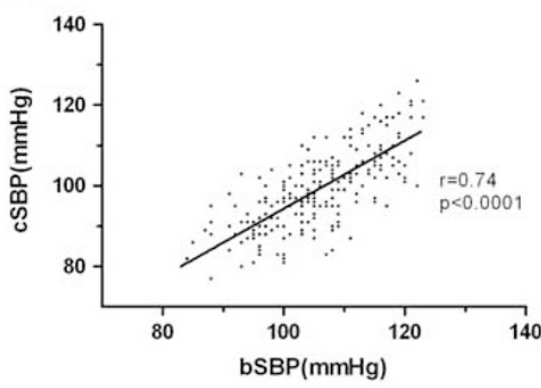

23-27weeks

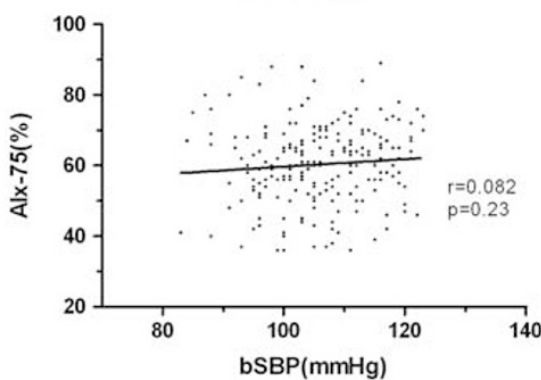

C

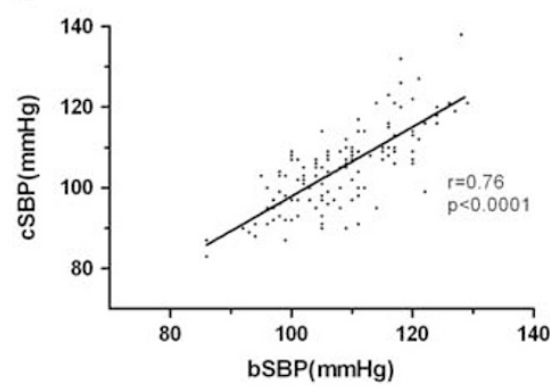

f

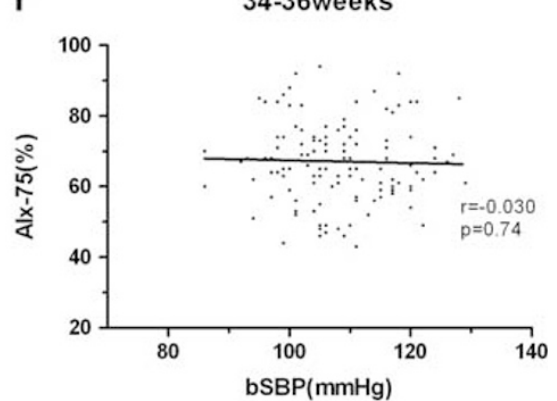

Figure 3 Relationships between brachial systolic blood pressure (bSBP) and central aortic systolic blood pressure (cSBP) (a-c) and augmentation index at heart rate of 75 b.p.m. (Alx-75) (d-f) values in the first trimester (12 to 14 weeks: a, d), in the late second trimester (23 to 27 weeks; b, e) and in the third trimester (35 to 37 weeks; $\mathbf{c}$, f). There were significant positive correlations between bSBP and cSBP in each trimester $(\mathbf{a} ; r=0.78, P<0.0001)$, $(\mathbf{b} ; r=0.74$, $P<0.0001$ ), (c; $r=0.76, P<0.0001$ ). In contrast, there were no correlations between bSBP and Alx-75 in each trimester in normal healthy pregnancies (d; $r=-0.0078, P=0.90)$, (e; $r=0.082, P=0.23)$, (f; $r=-0.030, P=0.74)$. 
The relationships between bSBP and cSBP, and AIx-75 in the first trimester (12 to 14 weeks), in the late second trimester (23 to 27 weeks) and in the third trimester (34 to 36 weeks) are shown in Figure 3. In contrast to cSBP, which was revealed to have significant correlations with bSBP throughout pregnancy (Figures 3a-c), AIx-75 did not show any correlations with bSBP in any trimesters in normal healthy pregnancies (Figures 3d-f).

Next, we examined the effects of maternal aging on bSBP, cSBP and AIx-75 in pregnant women less than 35 years old ( $n=604$; mean age: $28.8 \pm 3.9)$ and more than 35 years old $(n=220$; mean age: $37.0 \pm 2.0)$. (Figure 4). The age of 35 years was arbitrarily chosen, as this age has been frequently referred to the 'threshold' of advanced maternal age. In contrast to bSBP, which shows similar values and trends among groups, pregnant women more than 35 years old showed significantly higher CSBP and AIx-75 values compared with pregnant women less than 35 years old throughout pregnancy.

Figure 5 shows the longitudinal data of bSBP, cSBP and AIx-75 for the 69 normal pregnant women who had measurements taken at 12 to 14, 23 to 27 and 34 to 36 weeks gestation, at a few days after delivery and at 4 weeks postpartum. In contrast to bSBP, the fall in both cSBP and AIx-75 in the second trimester and the rise in both parameters after delivery were evident.

\section{DISCUSSION}

With the largest cohort of normal pregnancies, we showed that normal pregnancy is associated with a significant reduction in CSBP and AIx75 , reaching its nadir in mid-pregnancy and rising towards term. Our findings support two recent studies, which addressed normal values of cSBP and AIx throughout pregnancy. ${ }^{19,20}$ Furthermore, we found that AIx-75, but not cSBP, was independent of bSBP throughout pregnancy. Our present data suggested that measurement of cSBP and AIx might offer additional information about maternal arterial compliance, which might be impaired in various obstetric complications such as preeclampsia. These parameters also allowed detection of significant age-related differences in maternal arterial compliance in healthy pregnant women.

Pulse wave analysis using applanation tonometry has been increasingly used for the non-invasive assessment of central blood pressure and arterial stiffness, ${ }^{6-11}$ and several studies have confirmed that cSBP and AIx obtained from pulse wave analysis of peripheral arteries, such as the radial artery, are closely correlated with invasively measured cSBP and AIx. ${ }^{22-26}$ In addition, AIx has been reported to correlate with an endothelial function in healthy ${ }^{27}$ and cardiovascular compromised subjects. ${ }^{28}$

In a clinical setting, these parameters have been reported to be superior to traditional brachial artery blood pressure measurements in predicting future cardiovascular events, and in evaluating the response to drug treatment. ${ }^{2-5,29}$

During pregnancy, the maternal circulation undergoes remarkable physiological adaptations. Normal pregnancy is associated with increased cardiac output, intravascular volume and a marked decrease in vascular resistance. ${ }^{30-33}$ It has been reported that in pregnant women bSBP is a little below the non-pregnant level, rising in late pregnancy. ${ }^{34,35}$ On the basis of our findings, both cSBP and AIx-75 might be more suitable parameters than bSBP for assessing pregnancy-related changes in arterial compliance. Although both cSBP and AIx-75 showed similar changes during pregnancy and postpartum, they appeared to have different physiological meanings based on their completely different correlations with bSBP during pregnancy. The absence of correlation between AIx-75 and bSBP in our study is consistent with a previous study in healthy subjects, ${ }^{36}$ suggesting that
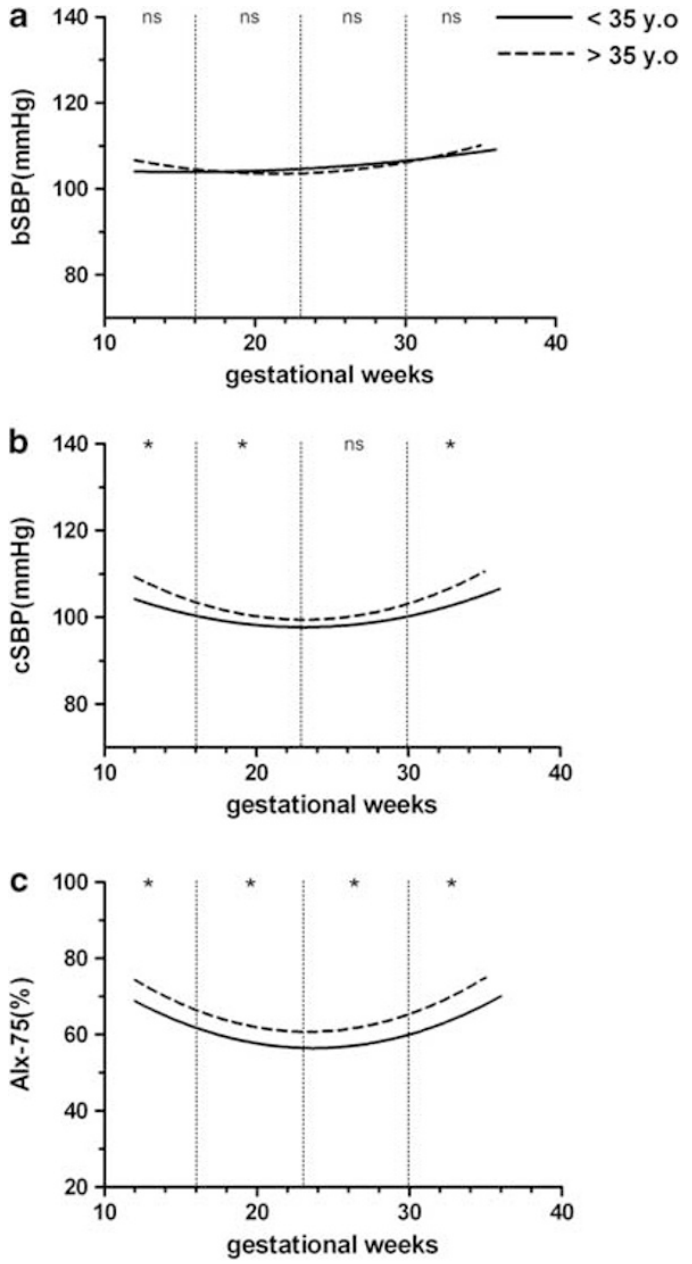

Figure 4 The trends and values of brachial systolic blood pressure (bSBP) (a), central aortic systolic blood pressure (cSBP) (b) and augmentation index at heart rate of 75 b.p.m. (Alx-75) (c) in pregnant women less than 35 years old (solid line, $n=604$ : mean age: $28.8 \pm 3.9$ ) and more than 35 years old (dashed line, $n=220$ : mean age: $37.0 \pm 2.0$ ). In contrast to bSBP, which shows similar values and trends among groups, pregnant women more than 35 years old (dashed line) showed higher CSBP and Alx-75 value compared with pregnant women less than 35 years old throughout pregnancy. * Significant difference among groups in each gestational week group $(P<0.05)$. ns, no significant difference among groups in each gestational week group.

both cSBP and AIx-75 represent different aspects of arterial compliance in healthy pregnant women as well.

Recently, several studies have increasingly reported impaired arterial compliance not only in pregnant women with preeclampsia, but also in gestational diabetes mellitus and type 2 diabetes, showing significantly increased cSBP and AIx in these population compared with normoglycemic controls. ${ }^{37}$ In addition, magnesium sulfate $\left(\mathrm{MgSO}_{4}\right)$ was recently reported to decrease AIx in women with preeclampsia, suggesting its possible mechanisms toward seizure prophylaxis. ${ }^{38}$ Although they did not mentioned cSBP values after magnesium sulfate administration, it might be possible that decreasing cSBP, which is a direct blood pressure to the brain, also contributes to seizure prophylaxis.

It has been reported that the age-related changes in cSBP and AIx are more prominent in younger individuals, whereas bSBP and pulse wave velocity increased significantly only after the age of $\sim 50$ years, 

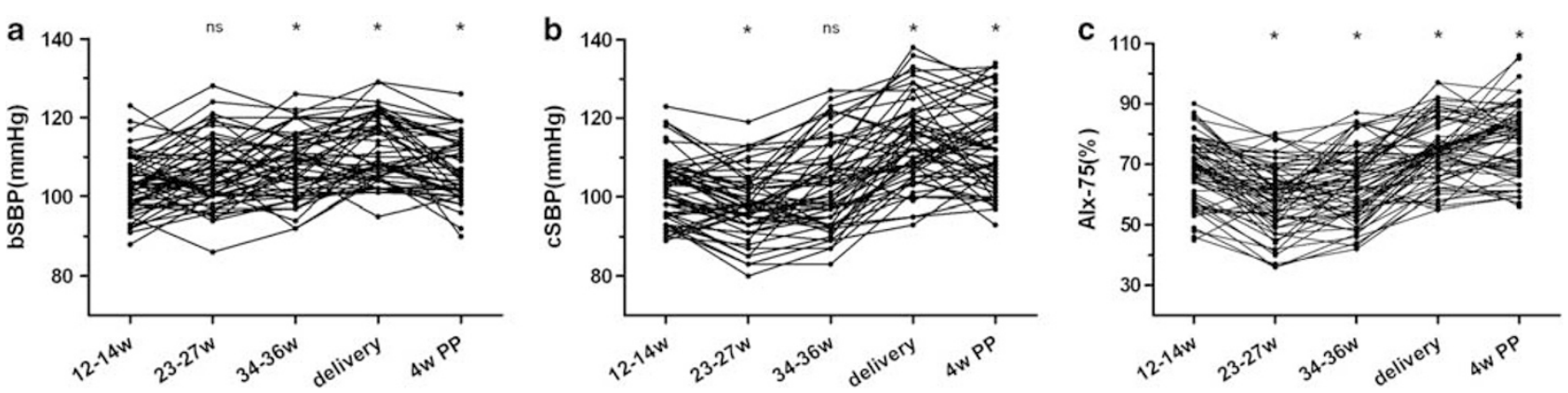

Figure 5 The longitudinal data of brachial systolic blood pressure (bSBP), central aortic systolic blood pressure (cSBP) and augmentation index at heart rate of 75 b.p.m. (Alx-75) for the 69 normal pregnant women who had measurements taken at 12 to 14,23 to 27 and 34 to 36 weeks of gestation, at a few days after delivery and at 4 weeks postpartum. In contrast to bSBP, the fall in both cSBP and Alx-75 in the second trimester and the rise in both parameters after delivery were evident. *Significant difference from measurements taken at 12 to 14 weeks gestation $(P<0.05)$. ns, no significant difference from measurements taken at 12 to 14 weeks gestation.

indicating that CSBP and AIx are earlier and more sensitive markers of aging especially in younger individuals. ${ }^{39}$ This report seems to support our finding of a significant increase in CSBP and AIx, but not bSBP, in pregnant women older than 35 years, and also seems to support incorporating these parameter into clinical practice as almost every pregnant woman is under 50 years. Pregnancy after age 35 is associated with an increased risk for maternal as well as neonatal complications, such as fetal growth disorders, maternal hypertensive disorders and gestational diabetes. ${ }^{40}$ Our finding of increased cSBP and AIx-75 in healthy pregnant women after age 35 might reflect one of the aspects of increased maternal and fetal adverse outcomes among these pregnancies.

In conclusion, this study established normal values for pulse wave analysis parameters in all three trimesters with gestation in the largest cohort of normal pregnancies, and indicated that pulse wave analysis might offer additional and independent information to conventional brachial blood pressure measurement. We hope that these data may be used as the basis for further investigation into the role of pulse wave analysis in the assessment, management and prediction of disorders, which might interfere with pregnancy-related cardiovascular adaptations.

\section{ACKNOWLEDGEMENTS}

This work was supported in part by a grant from the Smoking Research Foundation.

1 Sibai BM, Stella CL. Diagnosis and management of atypical preeclampsia-eclampsia. Am J Obstet Gynecol 2009; 200: 481.e1-7.

2 Williams B, Lacy PS, Thom SM, Cruickshank K, Stanton A, Collier D, Hughes AD, Thurston H, O'Rourke M. Differential impact of blood pressure-lowering drugs on central aortic pressure and clinical outcomes: principal results of the Conduit Artery Function Evaluation (CAFE) study. Circulation 2006; 113: 1213-1225.

3 Roman MJ, Devereux RB, Kizer JR, Lee ET, Galloway JM, Ali T, Umans JG, Howard BV. Central pressure more strongly relates to vascular disease and outcome than does brachial pressure: the Strong Heart Study. Hypertension 2007; 50: 197-203.

4 Chirinos JA, Zambrano JP, Chakko S, Veerani A, Schob A, Willens HJ, Perez G, Mendez AJ. Aortic pressure augmentation predicts adverse cardiovascular events in patients with established coronary artery disease. Hypertension 2005; 45: 980-985.

5 London GM, Blacher J, Pannier B, Guérin AP, Marchais SJ, Safar ME. Arterial wave reflections and survival in end-stage renal failure. Hypertension 2001; 38: 434-438.

6 Kelly R, Hayward CS, Avolio A, O'Rourke MF. Non-invasive registration of the arterial pressure pulse waveform using high-fidelity applanation tonometry. J Vasc Med Biol 1989; 1 : 142-149.

7 Nichols WW. Clinical measurement of arterial stiffness obtained from noninvasive pressure waveforms. Am J Hypertens 2005; 18: 3S-10S.

8 Nichols WW, Singh BM. Augmentation index as a measure of peripheral vascular disease state. Curr Opin Cardiol 2002; 17: 543-551.

9 Tomiyama H, Yamashina A. Non-invasive vascular function tests: their pathophysiological background and clinical application. Circ J 2010; 74: 24-33.
10 Ghiadoni L, Bruno RM, Stea F, Virdis A, Taddei S. Central blood pressure, arterial stiffness, and wave reflection: new targets of treatment in essential hypertension. Curr Hypertens Rep 2009; 11: 190-196.

11 Safar ME. Arterial aging-hemodynamic changes and therapeutic options. Nat Rev Cardiol 2010; 7: 442-449.

12 Rönnback M, Lampinen K, Groop PH, Kaaja R. Pulse wave reflection in currently and previously preeclamptic women. Hypertens Pregnancy 2005; 24: 171-180.

13 Oyama-Kato M, Ohmichi M, Takahashi K, Suzuki S, Henmi N, Yokoyama Y, Kurachi H. Change in pulse wave velocity throughout normal pregnancy and its value in predicting pregnancy-induced hypertension: a longitudinal study. Am J Obstet Gynecol 2006; 195: 464-469.

14 Kaihura C, Savvidou MD, Anderson JM, McEniery CM, Nicolaides KH. Maternal arterial stiffness in pregnancies affected by preeclampsia. Am J Physiol Heart Circ Physiol 2009; 297: H759-H764.

15 Avni B, Frenkel G, Shahar L, Golik A, Sherman D, Dishy V. Aortic stiffness in normal and hypertensive pregnancy. Blood Press 2010; 19: 11-15.

16 Khalil A, Jauniaux E, Harrington K. Antihypertensive therapy and central hemodynamics in women with hypertensive disorders in pregnancy. Obstet Gynecol 2009; 113: 646-654.

17 Khalil AA, Cooper DJ, Harrington KF. Pulse wave analysis: a preliminary study of a novel technique for the prediction of pre-eclampsia. BJOG 2009; 116: 268-276.

18 Yinon Y, Kingdom JC, Odutayo A, Moineddin R, Drewlo S, Lai V, Cherney DZ, Hladunewich MA. Vascular dysfunction in women with a history of preeclampsia and intrauterine growth restriction: insights into future vascular risk. Circulation 2010; 122: $1846-1853$

19 Macedo ML, Luminoso D, Savvidou MD, McEniery CM, Nicolaides KH. Maternal wave reflections and arterial stiffness in normal pregnancy as assessed by applanation tonometry. Hypertension 2008; 51: 1047-1051.

20 Khalil A, Jauniaux E, Cooper D, Harrington K. Pulse wave analysis in normal pregnancy: a prospective longitudinal study. PLoS One 2009; 4: e6134.

21 Funada J, Takata Y, Hashida H, Matsumoto Y, Sato S, Hiasa G, Inoue K, Higaki J, Okayama $\mathrm{H}$. Dysfunctional central hemodynamic regulation after daily meal intake in metabolic syndrome. Atherosclerosis 2010; 210: 268-273.

22 Takazawa K, Kobayashi H, Shindo N, Tanaka N, Yamashina A. Relationship between radial and central arterial pulse wave and evaluation of central aortic pressure using the radial arterial pulse wave. Hypertens Res 2007; 30: 219-228.

23 Kohara K, Tabara Y, Oshiumi A, Miyawaki Y, Kobayashi T, Miki T. Radial augmentation index: a useful and easily obtainable parameter for vascular aging. Am J Hypertens 2005; 18: 11S-14S

24 Millasseau SC, Patel SJ, Redwood SR, Ritter JM, Chowienczyk PJ. Pressure wave reflection assessed from the peripheral pulse: is a transfer function necessary? Hypertension 2003; 41: 1016-1020.

25 Sugawara J, Komine H, Hayashi K, Maeda S, Matsuda M. Relationship between augmentation index obtained from carotid and radial artery pressure waveforms. $J$ Hypertens 2007; 25: 375-381.

26 Melenovsky V, Borlaug BA, Fetics B, Kessler K, Shively L, Kass DA. Estimation of central pressure augmentation using automated radial artery tonometry. $J$ Hypertens 2007; 25: 1403-1409.

27 McEniery CM, Wallace S, Mackenzie IS, McDonnell B, Yasmin, Newby DE, Cockcroft JR, Wilkinson IB. Endothelial function is associated with pulse pressure, pulse wave velocity, and augmentation index in healthy humans. Hypertension 2006; 48: 602-608.

28 Soga J, Nakamura S, Nishioka K, Umemura T, Jitsuiki D, Hidaka T, Teragawa H, Takemoto H, Goto C, Yoshizumi M, Chayama K, Higashi Y. Relationship between augmentation index and flow-mediated vasodilation in the brachial artery. Hypertens Res 2008; 31: 1293-1298.

29 Hashimoto J, Imai Y, O'Rourke MF. Monitoring of antihypertensive therapy for reduction in left ventricular mass. Am J Hypertens 2007; 20: 1229-1233.

30 Mashini IS, Albazzaz SJ, Fadel HE, Abdulla AM, Hadi HA, Harp R, Devoe LD. Serial noninvasive evaluation of cardiovascular hemodynamics during pregnancy. Am J Obstet Gynecol 1987; 156: 1208-1213. 
31 Robson SC, Hunter S, Boys RJ, Dunlop W. Serial study of factors influencing changes in cardiac output during human pregnancy. Am J Physiol 1989; 256: H1060-H1065.

32 Duvekot JJ, Cheriex EC, Pieters FA, Menheere PP, Peeters LH. Early pregnancy changes in hemodynamics and volume homeostasis are consecutive adjustments triggered by a primary fall in systemic vascular tone. Am J Obstet Gynecol 1993; 169: 1382-1392.

33 Mabie WC, DiSessa TG, Crocker LG, Sibai BM, Arheart KL. A longitudinal study of cardiac output in normal human pregnancy. Am J Obstet Gynecol 1994; 170: 849-856.

34 Christianson RE. Studies on blood pressure during pregnancy. I. Influence of parity and age. Am J Obstet Gynecol 1976; 125: 509-513.

35 Moutquin JM, Rainville C, Giroux L, Raynauld P, Amyot G, Bilodeau R, Pelland N. A prospective study of blood pressure in pregnancy: prediction of preeclampsia. $A m \mathrm{~J}$ Obstet Gynecol 1985; 151: 191-196.
36 Yasmin, Brown MJ. Similarities and differences between augmentation index and pulse wave velocity in the assessment of arterial stiffness. QJM 1999; 92: 595-600.

37 Savvidou MD, Anderson JM, Kaihura C, Nicolaides KH. Maternal arterial stiffness in pregnancies complicated by gestational and type 2 diabetes mellitus. Am J Obstet Gynecol 2010; 203: 274.e1-7.

38 Rogers DT, Colon M, Gambala C, Wilkins I, Hibbard JU. Effects of magnesium on central arterial compliance in preeclampsia. Am J Obstet Gynecol 2010; 202 : 448.e1-8.

39 McEniery CM, Yasmin, Hall IR, Qasem A, Wilkinson IB, Cockcroft JR. Normal vascular aging: differential effects on wave reflection and aortic pulse wave velocity: the AngloCardiff Collaborative Trial (ACCT). J Am Coll Cardiol 2005; 46: 1753-1760.

40 Cunningham FG, Leveno KJ, Bloom SL, Hauth JC, Rouse DJ, Spong CY. Williams Obstetrics 23rd edn. McGraw-Hill Companies, Inc: New York, 2010, p.180-181. 